\title{
The effects of practice on mechanisms of attention
}

\author{
STEVEN P. TIPPER, THOMAS EISSENBERG, and BRUCE WEAVER \\ McMaster University, Hamilton, Ontario, Canada
}

\begin{abstract}
It is an open issue whether many of the effects observed in studies of selective attention are stable over extended periods of practice. Subjects are often placed in novel situations, and data is collected for 30-60 min. The results of such experiments are important, of course, because they determine models of attention. We examined three effects over 4 days: distractor interference, negative priming, and a form of response inhibition. The first two effects did not interact with practice, which suggests that the underlying mechanisms, such as inhibition of distractors, were stable. Conversely, the response inhibition effect did change with practice, but we suggest that the proportion of cued to uncued trials used in this study enabled conscious strategies to override the usual automatic inhibitory effects.
\end{abstract}

Mechanisms of attention are required to achieve coherent perceptual-motor interactions with complex environments. Many objects can afford simultaneous actions, but for most biological goals, such as capture of prey, action has to be directed selectively to only one object at a time.

The difficulty of selection can be observed experimentally through interference effects. Such effects as those reported by Stroop (1935) and Eriksen and Eriksen (1974) demonstrate that irrelevant stimuli are analyzed and compete with the target stimulus for the control of action, usually resulting in increased reaction times (RTs). For example, RT to name the color of the ink in which a word is written is slower if the word is a conflicting color name than if it is some word unrelated to color.

Interestingly, although interference effects can be substantial, selection is efficient, with few inappropriate responses to the irrelevant distractors. It has been proposed that inhibition of the internal representations of the distractors is one of the mechanisms of selection that prevent inappropriate responses (Neill, 1977; Tipper, 1985). Such inhibition has been observed by means of a priming procedure that is based on the following logic: If the internal representations of a distracting stimulus are associated with inhibition during both selection and response toward a target, then processing a subsequent stimulus requiring similar representations to those previously inhibited will be impaired. For example, if the distracting picture of a dog was inhibited during selection of a target, then the processing of that same picture of a dog shortly afterward will be impaired. Such a result has been observed in numerous situations and has been termed negative priming (Tipper, 1985).

In this latter situation, inhibitory mechanisms prevent an irrelevant stimulus from diverting action from a simul-

This research was supported by a Canadian Natural Science and Engineering Research Council grant awarded to Steve Tipper. Requests for reprints should be sent to Steve Tipper, Department of Psychology, McMaster University, Hamilton, ON, Canada L8S 4K1. taneously present target stimulus. However, in some situations, inhibitory mechanisms must prevent action toward a stimulus that is the focus of attention. Inhibition of action to an attended stimulus can again be observed by using a priming procedure. Thus, when a single stimulus is presented to which subjects must not respond, the inhibition associated with the stimulus will be observed through impaired response to a subsequent stimulus requiring the internal representations previously inhibited. We can consider this a form of response inhibition. ${ }^{1}$

In many experiments that purport to study selective attention, experimentally naive subjects have minimal experience with the task. That is, a novel situation is encountered for usually about $1 \mathrm{~h}$ or less. Clearly, there is little opportunity to achieve the level of experience we possess in many of our interactions with the real world. Therefore, there is the possibility that the mechanisms of attention under study are mainly utilized during initial encounters with an unusual situation. When familiarity increases, however, attentional selectivity may be achieved by quite different mechanisms.

Moreover, many studies of selective attention use filtering tasks, in which a target is selected by some physical cue, such as location, and the overt response is to identify the object, such as in naming pictures-that is, selectwhere-respond-what tasks. We have argued elsewhere that many perceptual-motor interactions are in fact opposite to this task (Tipper, Brehaut, \& Driver, 1990). Targets are often selected by category, and action is based on some physical property, such as location (e.g., reaching for a stimulus)-that is, select-what-respond-where tasks.

This study takes both of these factors into account. First, the select-what-respond-where task is used, and, second, the effects of practice on selective attention are examined. The subjects participated in the same task for 1-h sessions over a period of 4 days. Whether interference, negative priming, and response inhibition remain stable over this period is the central concern. 


\section{METHOD}

\section{Subjects}

The subjects were 12 undergraduates (4 males) enrolled in an introductory psychology course at McMaster University. They received course credit and \$8 for their participation.

\section{Apparatus and Stimuli}

All stimuli were presented on an Apple Ile computer equipped with a monochrome (green) monitor and joystick. The center of the monitor was marked with a small white circle affixed to the screen. Four white semicircular pieces of paper were affixed approximately $3 \mathrm{~cm}$ away from the center point and were located above, below, to the right, and to the left of the central fixation. These positions specified where the target and distractor could emerge.

\section{Procedure}

The subjects were seated in front of the computer and were asked to read the instructions that appeared on the screen. These instructions identified $O$ as the target and + as the distractor. A target, a distractor, or both could emerge from behind one of the four locations marked on the screen, although only one stimulus could occupy any one location. The subjects were required to move the joystick in the same direction as the location of the target. This joystick movement produced a line on the screen emanating from the center in the direction of the joystick movement. $^{2}$

To emphasize the importance of both speed and accuracy, a point system was instituted. Scores for each correct response ranged from 0 to 25, with higher scores corresponding to faster RTs. A correct (no response) distractor-only trial yielded 0 points, and 10 points were deducted for each incorrect response (i.e., responding to a nontarget location). Scores were reported to the subject after every two response opportunities, and a cumulative score was maintained throughout each block of trials (see below). This scoring system was explained to all subjects.

The subjects initiated each trial by pressing a button located on the upper left of the joystick box. Following a 1,500-msec pause, the first of two displays (the prime) appeared for $200 \mathrm{msec}$. The target and/or distractor appeared partially occluded for $50 \mathrm{msec}$, fully visible for $100 \mathrm{msec}$, and then partially occluded for another $50 \mathrm{msec}$. After the prime display, the screen went blank, and the subject was required either to respond if a target had appeared or to make no response if a target had not appeared. If no response was necessary, there was a 500-msec pause. After each prime response (or 500-msec pause), there was a 350msec response-stimulus-interval (RSI), following which a second display (the probe) was presented in the same manner as the prime. After the subject's response to the probe, the cumulative score and scores for the most recent prime and probe were presented, and the subject was prompted to initiate another trial.

The subjects participated in 12 blocks of trials, with each block consisting of a practice session and an experimental session. Sessions ended when the subjects reached a criterion level of correct responses; for all 12 practice sessions, the criterion was 2 correct per condition, and for the experimental sessions, it was 20 correct per condition. The subjects completed 3 sessions per day for 4 consecutive days.

\section{Design}

Each trial consisted of two displays: a prime and a probe. There were three types of prime displays: target-alone, target-and-distractor, and distractor-alone (see Figure 1). Interference is the difference between target-alone and target-and-distractor prime displays and is manifested in higher RTs and error rates in the target-and-distractor condition.

The probe displays of interest are those that follow target-and-distractor primes and distractor-alone primes. Two different probe conditions followed target-and-distractor primes: control (CTRL), and ignored repetition (IR). In the CTRL condition, the probe target and the distractor occupied the two positions that were unused in the prime. In the IR condition, the probe target appeared in the location that had been occupied by the prime distractor, and the probe distractor appeared in one of the two positions that had been unoccupied during the prime (see Figure 1). Negative priming would be indicated by greater RTs and error rates in the IR condition.

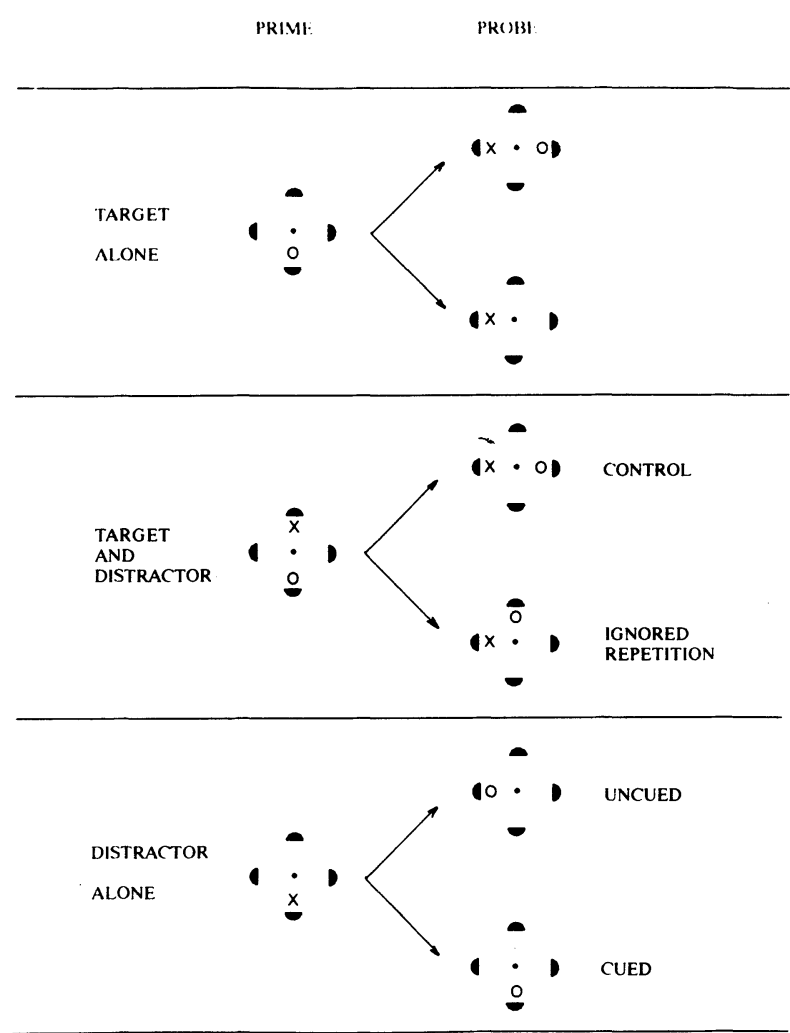

Figure 1. Examples of the prime and probe displays. The prime display consists of target-alone, target-and-distractor, and distractoralone conditions. The difference between the target-alone and the target-and-distractor conditions reflects the interference effect. The probe display contains the control, ignored repetition, uncued, and cued conditions. The contrast between control and ignored repetition reflects negative priming; the contrast between uncued and cued reflects response inhibition. The two probe conditions following target-alone primes were of no theoretical interest and were not analyzed.

In the uncued and cued probe conditions that followed distractor-alone primes, only a target was presented. In the uncued condition, that target appeared in one of the three locations that had been unoccupied during the prime. In the cued condition, the target appeared in the same location as the prime distractor. A response inhibition effect would be indicated by greater RTs and error rates in the cued condition.

The effects of interference, negative priming, and response inhibition were all tested within-subjects over 12 blocks of trials ( 3 blocks a day for $\mathbf{4}$ consecutive days). To assess the effects of the subjects' awareness on interference, negative priming, and response inhibition, half of the subjects $(n=6)$ were debriefed as to the nature of the experiment before their final block of trials, whereas the rest were debriefed after their final block.

\section{RESULTS AND DISCUSSION}

\section{Interference}

Figure 2A displays the means of all of the subjects' median RTs by block for the target-only and the target-anddistractor conditions. ${ }^{3}$ The 11 blocks were analyzed by using a 2 (interference) $\times 11$ (practice) within-subject analysis of variance (ANOVA). There was a significant effect of interference $[F(1,11)=35.15, p<.001]$. As 

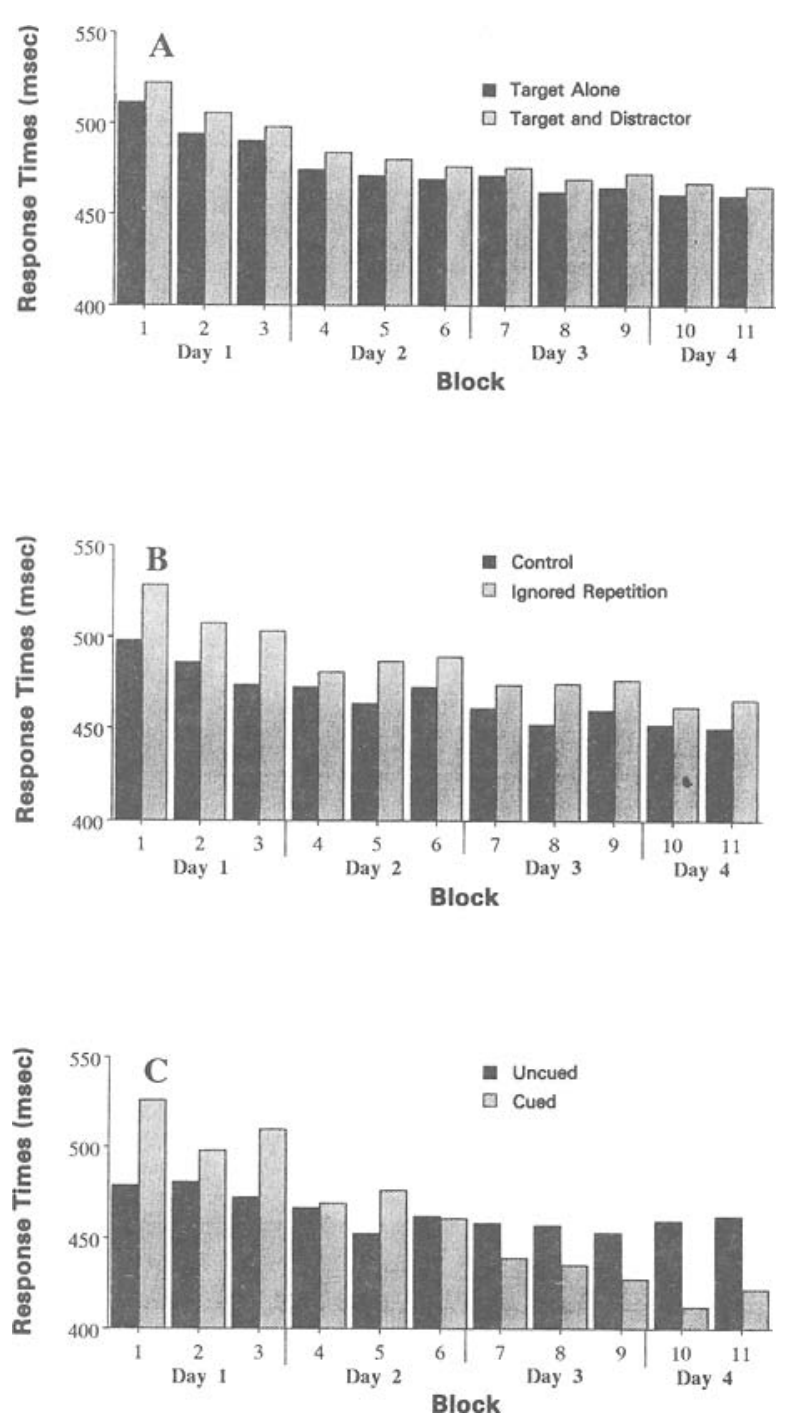

Figure 2. Mean response times for experimental conditions and blocks. Panels A, B, and C represent interference, negative priming, and response inhibition, respectively.

predicted, RTs were longer when a distractor was present. A significant effect of practice was also obtained $[F(10,110)$ $=9.62, p<.0001]$ when RTs became faster with increased practice. Most importantly, there was no interaction between practice and interference effects $[F(1,110)<1]$. This latter, nonsignificant interaction indicates that the interfering effects of an irrelevant stimulus remain constant even after extended practice, indicating that selection efficiency remains fairly stable.

Table 1 indicates error rates. A two-way within-subject ANOVA yielded no significant effects of interference, practice, or interaction between them.

\section{Negative Priming}

Figure 2B displays by block the means of all of the subjects' median RTs for the CTRL and IR conditions. The main effect of negative priming was highly significant $[F(1,11)=53.95, p<.0001]:$ RTs were longer for IR trials. As with the interference effects, the effect of practice was also significant $[F(1,110)=7.11, p<.0001]$. Again of most importance, there was no evidence for an interaction between practice and negative priming $[F(10,110)=1.21$, n.s. $]$. Clearly, there appears to be no effect of practice on inhibitory mechanisms of selective attention.

Error rates are displayed in Table 1. There was no effect of negative priming, a significant effect of practice in line with the RTs $[F(10,110)=4.82, p<.001]$, and no interaction.

\section{Response Inhibition}

Figure 2C displays by block the means of all of the subjects' median RTs for the uncued and cued conditions. A two-way within-subject ANOVA revealed no effect of response inhibition $[F(1,11)<1]$, a significant effect of practice $[F(1,110)=10.53, p<.01]$, and a highly significant interaction between response inhibition and practice $[F(10,110)=12.02, p<.0001]$. As can be seen in Figure 2C, inhibition effects are initially observed, but these are replaced by facilitatory effects by Block 7 .

This response inhibition effect is affected by practice in a qualitatively different way from that of negative priming. The former changes from inhibition to facilitation with increased experience with the task, whereas the latter remains inhibitory. The facilitatory response inhibition can be accounted for by the contingencies of the conditions. For probe displays following distractor-alone primes, there was a $50 \%$ probability that the target would appear in the same location as the prime distractor and only a $16.7 \%$ probability of the target appearing in each of the three uncued locations. Subjects are probably discovering this relationship and are using the no-go prime to predict the locus of the subsequent probe.

We suggest that this strategy is possible because attention is directed toward the prime. It is interesting to note that a biased probability between prime and probe trials also exists for negative priming. That is, after presentation of a target-and-distractor prime, there is a $50 \%$ probability that the probe target will be in the same location

Table 1

Mean Percentage Error Rates

\begin{tabular}{|c|c|c|c|c|c|c|}
\hline \multirow[b]{3}{*}{ Block } & \multicolumn{6}{|c|}{ Phenomenon } \\
\hline & \multicolumn{2}{|c|}{ Interference } & \multicolumn{2}{|c|}{$\begin{array}{c}\text { Negative } \\
\text { Priming }\end{array}$} & \multicolumn{2}{|c|}{$\begin{array}{l}\text { Response } \\
\text { Inhibition }\end{array}$} \\
\hline & T Alone & $T$ and $\mathrm{D}$ & Control & IR & Uncued & Cued \\
\hline 1 & 0.38 & 0.55 & 3.15 & 3.63 & 0.00 & 1.45 \\
\hline 2 & 0.16 & 0.87 & 2.20 & 4.83 & 1.44 & 2.14 \\
\hline 3 & 0.19 & 0.75 & 0.38 & 0.76 & 1.13 & 0.38 \\
\hline 4 & 0.19 & 0.55 & 1.52 & 2.26 & 0.00 & 0.00 \\
\hline 5 & 0.19 & 0.56 & 0.00 & 0.75 & 1.51 & 0.38 \\
\hline 6 & 0.38 & 0.19 & 0.38 & 0.75 & 0.72 & 0.35 \\
\hline 7 & 0.35 & 0.92 & 0.75 & 1.45 & 1.51 & 0.35 \\
\hline 8 & 0.38 & 0.56 & 0.00 & 0.38 & 1.10 & 0.38 \\
\hline 9 & 0.19 & 0.56 & 1.13 & 0.75 & 0.38 & 0.38 \\
\hline 10 & 0.36 & 0.18 & 1.82 & 1.82 & 1.07 & 1.48 \\
\hline 11 & 0.19 & 0.56 & 1.48 & 0.38 & 0.00 & 0.00 \\
\hline
\end{tabular}

Note-T $=$ target, $\mathbf{D}=$ distractor. 
as the prime distractor, whereas there is only a $25 \%$ probability that the probe target will appear in each of the other possible positions. It is possible that this contingency in negative priming trials is not detected and utilized by subjects because the prime is ignored. Support for this view was gained from analysis of Block 12 , which was tested at the end of Day 4. The subjects were divided into two groups. One group was informed of the various conditions tested; the other group remained uninformed. There was a significant interaction between negative priming and knowledge $[F(1,10)=6.16, p<.05]$ such that the uninformed subjects continued to produce negative priming $(-19 \mathrm{msec})$, but those made aware of the conditions produced a small facilitatory priming effect $(14 \mathrm{msec})$. No such interaction was observed for response inhibition, which would be predicted if the subjects were already aware of the conditions.

\section{CONCLUSION}

This experiment suggests that the mechanisms of selective attention remain stable over increasing levels of practice. That is, at the end of the experiment, 1,452 trials $(1,452 \times 2=2,904$ individual prime and probe events) had been completed. Even though performance improved greatly, selection efficiency remained relatively stable, and there was evidence that inhibition of distractors was a mechanism used throughout the experiment. This pattern of results supports that obtained by Tipper, Weaver, Kirkpatrick, and Lewis (in press). Conversely, response inhibition was influenced by the level of experience with the task. In this case, the initial inhibition was replaced by subsequent facilitatory priming effects.

A second unpublished experiment requiring selection based on categorical properties (target $=\mathrm{P}$ or $\mathrm{B}$, distractor $=8$ or 9) rather than simple features (target $=0$, distractor $=+$ ) produced essentially the same results. That is, both significant interference and negative priming effects were obtained, and they did not interact with practice over the 4 days of training. Once again, however, response inhibition did interact with practice.

Recent research by Cheesman, Graf, and Bourassa (1990) has questioned whether negative priming is a stable mechanism. Using a Stroop color-word task, they report that the effect disappears after about 300 trials. It is therefore possible that the stability of attentional mechanisms is determined by task demands. It is certainly the case that Stroop negative priming effects have properties that differ with the effects observed in other experimental procedures. For example, Lowe (1979) demonstrated that Stroop negative priming effects were influenced by the proportion of the conditions in the experiment. We have not found this in other procedures, and this may explain conflicting findings, such as the observation of Stroop negative priming by Neill (1977) and Neill and Westberry (1987) after 1,000 trials.

Finally, it is an open question whether further practice would have resulted in a change in selection strategies. Few experiments in cognitive psychology examine performance after thousands of trials. But whether, for example, inhibitory mechanisms of selection are always utilized is clearly a relevant issue.

\section{REFERENCES}

Cheesman, J., Graf, P. L., \& Bourassa, D. A. (1990, November). Attentional inhibition: General mechanisms or task effect? Paper presented at the 31st Annual Meeting of the Psychonomic Society, New Orleans.

Eriksen, B. A., \& Eriksen, C. W. (1974). Effects of noise letters upon the identification of a target letter in a nonsearch task. Perception \& Psychophysics, 16, 143-149.

LoGAN, G. D. (1985). On the ability to inhibit simple thoughts and actions: II. Stop-signal studies of repetition priming. Journal of Experimental Psychology: Leaming, Memory, \& Cognition, 11, 675-691.

LowE, D. G. (1979). Strategies, context, and the mechanism of response inhibition. Memory \& Cognition, 7, 382-389.

NEILL, W. T. (1977). Inhibition and facilitation processes in selective attention. Journal of Experimental Psychology: Human Perception \& Performance, 3, 444-450.

NeILL, W. T., WESTBerRY, R. L. (1987). Selective attention and the suppression of cognitive noise. Journal of Experimental Psychology: Learning, Memory, \& Cognition, 13, 327-334.

PoSNer, M. I., \& CoHEN, Y. A. (1984). Components of visual orienting. In H. Bouma \& D. G. Bouwhuis (Eds.), Attention and performance $X$ (pp. 531-554). Hillsdale, NJ: Erlbaum.

STROOP, J. R. (1935). Studies of interference in serial verbal reactions. Journal of Experimental Psychology, 18, 643-662.

TIPPER, S. P. (1985). The negative priming effect: Inhibitory effects of ignored primes. Quarterly Journal of Experimental Psychology, 37A, 571-590.

TIPPER, S. P., BrehaUt, J. C., \& DRIVER, J. (1990). Selection of moving and static objects for the control of spatially directed action. Journal of Experimental Psychology: Human Perception \& Performance, 16, 492-504.

TiPPER, S. P., Weaver, B., KirkPatrick, J., \& LeWIs, S. (in press). Inhibitory mechanisms of attention: Locus, stability, and relationship with distractor interference effects. British Journal of Psychology.

\section{NOTES}

1. This effect is similar to others reported in the literature. For example, it bears some resemblance to the inhibition of return reported by Posner and Cohen (1984) and the stop-signal inhibition reported by Logan (1985).

2. This procedure attempted to make the experiment more like a game. The task was originally designed for studies with children in the first grade. Therefore, it was described in terms of a "space invaders" game, in which subjects had to shoot the target $O$ (the bad guy) as fast as possible, while avoiding shooting the distractor + (the good guy). The scoring system, about to be described, also attempted to encourage high levels of speed and accuracy in the context of gaining points in the game.

3. Although the clock card used to time responses has a resolution of about $1000 \mathrm{~Hz}$, raw RTs are accurate only to about $45 \mathrm{msec}$ because of the rate at which the CPU checks for joystick responses when the AppleSoft Basic PADDLE ( ) function is used. Despite this relatively low resolution, we are confident that we have meaningful data for two reasons: First, having low resolution makes it more likely that we will fail to detect a real effect (i.e., commit a Type II error), and, second, we have previously demonstrated that negative priming persists over 3 days of practice when RTs are measured by using a voice key, which does not have the same sampling problem as the joystick (see Tipper, Weaver, Kirkpatrick, \& Lewis, in press). 